\title{
Effectiveness of PAM-4 Line Coding in Triplication-based Error Correction Codes under Harsh Electromagnetic Disturbances
}

\author{
Jonas Van Waes \\ Department of Computer Science \\ KU Leuven Campus Bruges \\ Bruges, Belgium \\ jonas.vanwaes@kuleuven.be \\ Dries Vanoost \\ Department of Electrical Engineering \\ KU Leuven Campus Bruges \\ Bruges, Belgium \\ dries.vanoost@kuleuven.be
}

\author{
Jens Vankeirsbilck \\ Department of Computer Science \\ KU Leuven Campus Bruges \\ Bruges, Belgium \\ jens.vankeirsbilck@kuleuven.be \\ Davy Pissoort \\ Department of Electrical Engineering \\ $K U$ Leuven Campus Bruges \\ Bruges, Belgium \\ davy.pissoort@kuleuven.be
}

\author{
Jonas Lannoo \\ Department of Electrical Engineering \\ KU Leuven Campus Bruges \\ Bruges, Belgium \\ jonas.1annoo@kuleuven.be
}

Jeroen Boydens

Department of Computer Science

KU Leuven Campus Bruges

Bruges, Belgium

jeroen.boydens@kuleuven.be

\begin{abstract}
In harsh electromagnetic environments, Error Detection and Correction Codes can be used to counteract transmission errors. However, the overhead introduced by Triplicationbased codes can be detrimental for the communication capabilities of the system. This paper assesses if a Pulse Amplitude Modulation with 4 symbols (PAM-4) is worth using in harsh electromagnetic environments. It is found that some systems can benefit from PAM-4 in terms of availability, but a significant trade-off in terms of False Negatives must be made. A False Negative arises when the Error Detection and Correction Code deems the received data valid, whilst it was corrupted during transmission.
\end{abstract}

Index Terms-EMI Resilience, Error Correction Codes, False Negatives, Line Coding

\section{INTRODUCTION}

Recent research assessed the effectiveness of different Error Correction Codes (ECCs) under harsh electromagnetic conditions for safety-critical applications [1]-[6]. This research specifically focused on the elimination of False Negatives from a communication channel. A False Negative arises when the Error Detection and Correction Code deems the received data valid, whilst it was corrupted during the transmission.

The channel itself is exposed to harsh, electromagnetic disturbances at a single frequency (e.g. radar). Even though the research succeeded in eliminating the False Negatives for certain disturbances, it left the system with a severe overhead. Multiple EDCCs were considered, such as Hamming Codes and Triplication. For 4-bit data, the overhead is $75 \%$ and $200 \%$, respectively. This paper will focus on reducing the

This work is supported by a research grant from the Flemish Agency for Innovation and Entrepreneurship (VLAIO) within the HBC.2017.0650 RESSIAR-MID/TransSIMS project.
Triplication overhead, as it is the most severe. As an example, straightforward Triplication is considered and is indicated in this paper as TEC: Triplication with Equal bit encoding with the bits placed $\underline{\text { Close together. }}$

The goal of this paper is to reduce the overhead whilst maintaining the same performance and effectiveness under the considered electromagnetic disturbances. Up until now, the research with Triplication made use of a Non-Return-toZero Level (NRZ-L) line encoding. In this paper, the PAM4 modulation replaces the NRZ-L. Note that NRZ-L is also known as PAM-2, since it uses two symbols.

\section{Simulation Setup}

The effect of continuous-wave EMI on Error Correction Codes (ECCs) has been detailed previously in [4] and [5]. For clarity and to assist the reader in understanding the remainder of this paper, the simulation setup is repeated here. Moreover, Section II-A is a repetition from Section III of [7] where the induced voltage calculation is elaborated.

Fig. 1 gives a conceptual overview of the experimental setup that will be considered in the remainder of this paper. This setup was previously used in [1] and [7]. The Device Under Test (DUT) comprises a Printed Circuit Board (PCB) with a single $50 \Omega$ micro-strip. One end of the micro-strip is driven with a $1 \mathrm{~V}$ power supply with a $50 \Omega$ output impedance. The other end of the micro-strip is terminated with a $50 \Omega$ load. The PCB itself is an FR4 substrate of $10 \mathrm{~cm}$ by $16 \mathrm{~cm}$ and has a thickness of $1.6 \mathrm{~mm}$, with a dielectric constant of 4.7. The trace is $3 \mathrm{~mm}$ wide and has a length of $5 \mathrm{~cm}$. The bottom of the PCB is covered with a ground plane.

A data producer generates data words, encodes them (either with or without an EDCC) and puts these code words onto 


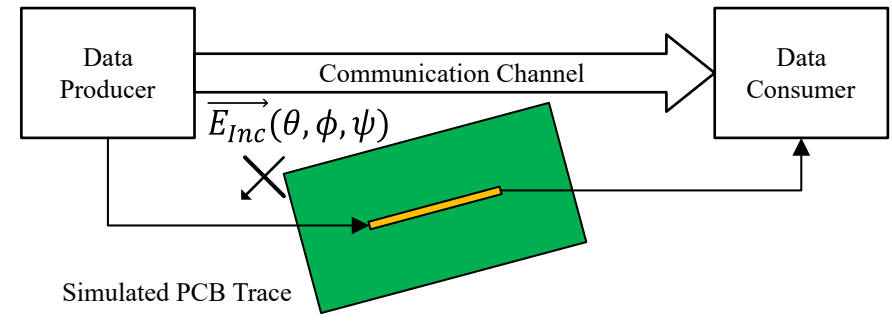

Fig. 1. Simulated Trace as Communication Channel [1]

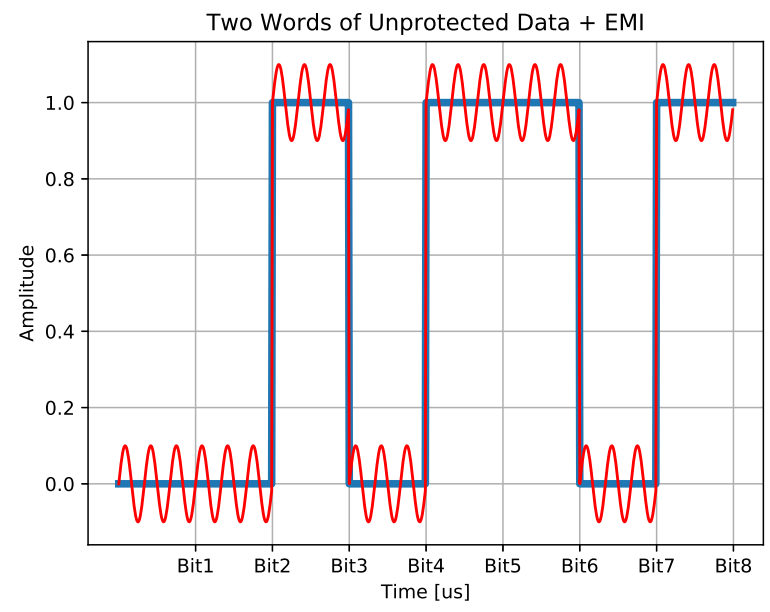

Fig. 2. The considered signals in the time domain. The blue line represents a transmitted bit stream. The red line is the received signal: the induced disturbance voltage added to the transmitted bit stream.

the communication channel (the PCB trace in this case). The channel then transmits the code words to the data consumer. The latter decodes the received code words to data words. The entire communication subsystem is subjected to an EMI disturbance, represented schematically in Fig. 1 by $E_{i n c}$. In order to check the effectiveness of the considered EDCCs, the decoded data word is compared to the original data word that was sent. A conceptual overview of the considered time signals can be seen in Fig. 2.

For this paper, the electromagnetic disruption under consideration is a single-frequency disturbance. The simulations themselves allow the implementation of a multi-frequency disturbance. However, this complicates the illustrative potential of the figures within this paper.

\section{A. Calculation of the Induced Voltages}

Calculating the induced voltages at the end of the microstrip due to incoming disturbances can be done using several methods. One of those methods is using a full-wave simulation for each possible way an EMI disturbance could disturb the DUT. This would require a huge amount of simulations and simulation time. Therefore, an efficient reciprocity-based algorithm is used. This method uses only one full-wave simulation per port. In each of these simulations, one port is excited and the far-field of the DUT is calculated. Using the far-field

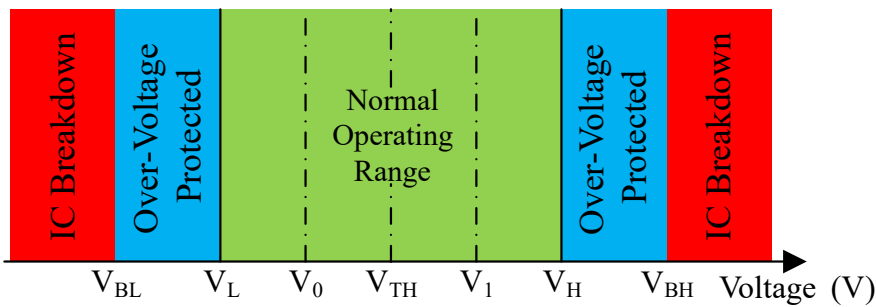

Fig. 3. Decoding Voltage Thresholds (Section II-B)

data and the reciprocity theorem, the induced voltages can be calculated on all ports as a post-processing step for whatever incoming EMI disturbance [8].

In addition, this paper considers the harsh EMI-environment to be a reverberating environment. Similar to the real world, a reverberation room environment consists out of a lot of reflections [7]. These conditions are created by using the plane wave integral representation for reverberation chambers as presented in [9].

This uses the work of [10] to obtain a statistical plane-wave representation of a reverberation room-like environment. The different parameters for the statistical distribution, which use spherical coordinates, are:

- The uniformly distributed azimuth angle $\phi$ in the range $[0,2 \pi]$

- The polar angle $\theta$ with an uniformly distributed $\cos (\theta)$ in the range $[-1,1]$;

- The uniformly distributed polarization angle $\psi$ in the range $[0, \pi]$;

- The uniformly distributed phase angle $\alpha$ in the range $[0,2 \pi]$.

The last step after superposing all random plane waves is to normalize the amplitude of each separate plane wave by $1 / N^{0.5}$ [8]. The amount of plane waves that are combined together could be represented as the amount of modes that are simultaneously excited in a real reverberating environment [10].

The plane waves that are combined are a set of $\mathrm{N}$ plane waves, each with random properties for the polar angle $\theta$, azimuth angle $\varphi$, polarization angle $\psi$ and phase angle $\alpha$. To represent the continuously changing nature of a reverberation environment, this simulation is done $\mathrm{M}$ times and each time a different set of $\mathrm{N}$ plane waves is chosen. For this study, $\mathrm{M}$ and $\mathrm{N}$ were chosen to be 10,000 and 200 , respectively.

\section{B. Over-voltage Protection}

A well-designed safety-critical system is assumed to be protectd against over-voltages at, e.g., the pins of a Integrated Circuits (ICs). Therefore, there are several thresholds to be considered when decoding a received voltage to a logic ' 1 ' or ' 0 ' bit value. This is schematically depicted in Fig. 3.

The normal operating voltage range goes from $V_{\mathrm{L}}$ to $V_{\mathrm{H}}$, whereas the over-voltage protection is activated between $V_{\mathrm{BL}}$ to $V_{\mathrm{L}}$ (low end) and between $V_{\mathrm{H}}$ to $V_{\mathrm{BH}}$ (high end). For voltages below $V_{\mathrm{BL}}$ or above $V_{\mathrm{BH}}$, the IC will break down. The 
latter is a situation which must be avoided as much as possible. In a well-designed safety-critical system, IC breakdown is avoided by the over-voltage protection mechanisms. These mechanisms clamp the voltage to a voltage within the blue region on Fig. 3, preferably well below $V_{\mathrm{BH}}$ or above $V_{\mathrm{BL}}$.

In the remainder of this paper, we assume that the system can actually detect and know that the over-voltage protection has been triggered, as described in [11]. This allows the initiation of the necessary steps to ensure overall safety is maintained. This state is called the Minimal Risk State (MRS). This can be the transitioning to a safe state, if such a state exists in the application at hand, or transitioning to a degraded mode in which only limited functionality is available.

\section{Fault Categorization}

Following the different voltage thresholds defined in Fig. 3, five distinct categories are considered after decoding. The categories are equal to the categories defined in [4], [5]:

- Error-free Data (EFD): The decoded data is correct and no error was detected during decoding. This is the normal, desired and expected behavior and is marked in green;

- Succesful Correction (SCO): The decoded data is correct, but only after the correction of the applied EDCC. The system knows that the data has been altered by the EDCC and that care must be taken in processing the data (e.g. transitioning to a degraded mode with limited functionality). However, this is still an acceptable category and is marked in yellow;

- Failed Correction (FCO): The decoded data is incorrect, despite an attempt of the EDCC to correct it. The system knows that the data has been altered by the EDCC and that care must be taken in processing the data (e.g. transitioning to a degraded mode with limited functionality). This category is undesired and marked in orange;

- False Negative (FN): The decoded data is incorrect, but the EDC did not even detect that an erroneous code word was received. Thus, the system incorrectly assumes that the received data word is valid for use, which could easily lead to (very) dangerous failures of the system. This scenario is detrimental to the overall system safety. This category should be avoided as much as possible and is therefore marked in red;

- Over-voltage Protected (OVP): The over-voltage protection is triggered and a well-designed safety-critical system induces an operation to ensure the overall system safety is maintained. This is a desired category and marked in blue.

An important focus of this paper is to study how the line coding affects the rate of False Negatives, indicated with the red category. For safety-critical systems, this red category of False Negatives is to be avoided because no countermeasures can be taken at this level. The system is unaware that the received data is incorrect, which could result in catastrophic failures.

For the fault categorization, we must assume that higher software layers can make the distinction between SCO and
FCO. This can be achieved by e.g. a Cyclic Redundancy Check (CRC). Although indistinguishable at this level, SCO and FCO have a separate severity level throughout the system, which is why it is separatly considered in this paper.

\section{Monte-Carlo-based Simulation Framework}

As stated above, the DUT is subjected to harsh EMI disturbances (being a superposition of a large number of plane waves at the same frequency $f_{\mathrm{EMI}}$ ). As we want to draw general conclusions, a Monte Carlo simulation is performed as presented in [2] and [3]. In this Monte Carlo simulation, the parameters of the incident plane waves are chosen randomly, while also statistically varying the time delay between the disturbance and the transmitted bits. Moreover, in our study, we swept the bit rate (denoted as $B R$ in all formulas and equations) from $1 \mathrm{Mbps}$ to $1500 \mathrm{Mbps}$ in steps of $1 \mathrm{Mbps}$, while $f_{\mathrm{EMI}}$ equalled ten specific values as noted in (1).

$$
\left\{\begin{aligned}
& f_{\mathrm{EMI}}=\{97,100,257,313,419,439 \\
&500,683,797,929,967\} \mathrm{MHz} \\
& B R \in[1,1500] \mathrm{Mbps} \mid \Delta B R=1 \mathrm{Mbps} \\
& E_{\text {inc }}= 400 \mathrm{~V} / \mathrm{m}
\end{aligned}\right.
$$

Previous experiments already showed a correlation between the ratio of $f_{\mathrm{EMI}}$ and $B R$ on the one hand, and the susceptibility to EMI on the other hand [1]-[3]. Therefore, ten frequencies (from which most are prime frequencies) were selected and the bit rate was swept. The goal was to explicitly uncover the correlation and influence of the bit rate to disturbance frequency ratio. Also, it is unfeasible to perform a full coverage of all possible frequencies, bit rates and incoming field strengths. This continuous state space is too wide to cover. Therefore, a subset has been chosen. Note that the fault injection model does consider all possible faults. However, no consideration of the actual likelihood of the disturbance occurring in the field have been implemented.

Since similar results were obtained for different incident field strengths and communication channel threshold voltages, the incident field strength is fixed to $400 \mathrm{~V} / \mathrm{m}$. This value is used for all examples considered in the remainder of this paper. Since the results of the Monte Carlo simulations are similar for each $f_{\mathrm{EMI}}$, the remainder of this paper only describes the results for $f_{\mathrm{EMI}}=683 \mathrm{MHz}$ (unless explicitly stated otherwise).

The last parameter to be considered is the type of line coding. Fig. 4 shows both NRZ-L and PAM-4 with their respective logic and voltage levels. As the figure shows, PAM4 combines two bits into one symbol (voltage level). PAM4 has much less noise margin between the various symbol levels. The noise margin between symbols for NRZ-L is 0.25 $\mathrm{V}$, whilst for PAM-4 the noise margin is reduced to $0.083 \mathrm{~V}$. This difference will have a major impact on the distribution of the fault categories.

\section{COMPARISON BETWEEN THE LINE CODINGS}

The influence of the reduced noise margin on the fault categorization between NRZ-L and PAM-4 is shown in Figs. 5 


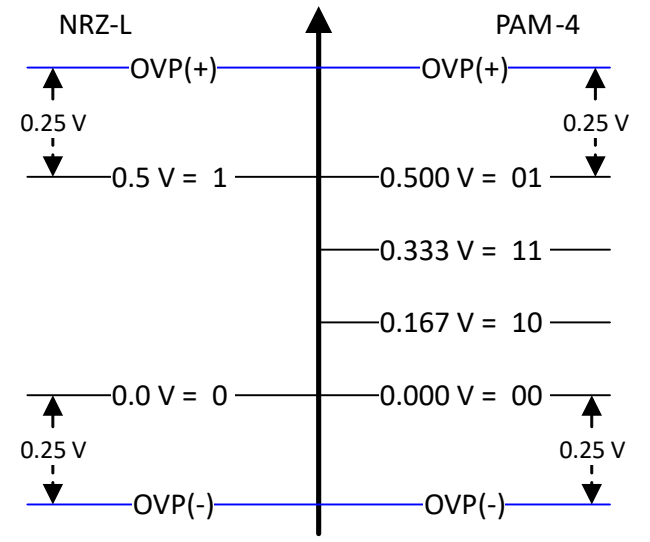

Fig. 4. Conversion from PAM-2 (NRZ-L) to PAM-4

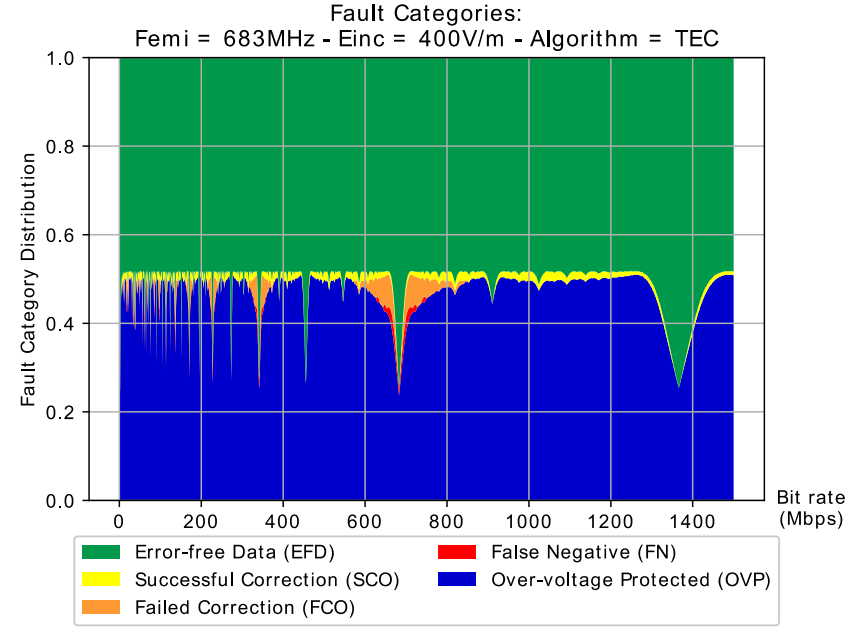

Fig. 5. Fault Categorization for NRZ-L encoded TEC

and 6. The differences between the fault categorizations for each line code will be discussed using the overall mean values of each category. The values are listed in Table I.

\section{A. Over-voltage Protected}

The OVP drops drastically from $46.64 \%$ for NRZ-L to a $33.32 \%$ for PAM-4. This observation has a twofold cause.

Firstly, the number of sampling points is halved. Whereas the NRZ-L triplication required 12 symbols (one symbol for one bit), triplication encoded with PAM-4 only requires 6

TABLE I

FAUlt CATEGORIZATION FOR BOTH LINE CODINGS (IN \%)

\begin{tabular}{llll}
\hline Category & NRZ-L & PAM-4 & Difference \\
\hline OVP & 46.64 & 33.32 & -13.32 \\
EFD & 50.64 & 10.02 & -40.62 \\
SCO & 01.44 & 21.93 & +20.49 \\
FCO & 01.05 & 34.57 & +33.52 \\
FN & 00.23 & 00.16 & -00.07 \\
Availability & 52.08 & 31.95 & -20.13 \\
Safe & 99.77 & 99.84 & +00.07 \\
\hline
\end{tabular}

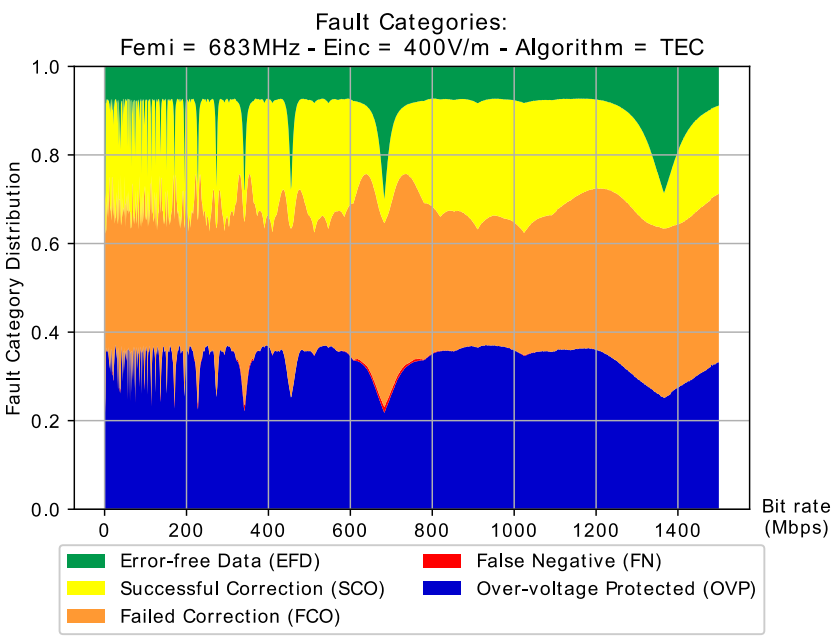

Fig. 6. Fault Categorization for PAM-4 encoded TEC

symbols (two bits for each symbol). This results in less chance of a sampling point triggering the OVPM.

The second reason for the drop in this category, is that the voltages for 11 and 10 have a larger distance to the overvoltage protection thresholds. For NRZ-L, a symbol change for one sampling point almost always results in an OVPMtrigger for another sampling point (low percentage of SCO and FCO). In contrast, the required voltage to flip a symbol in PAM-4 (about $0.083 \mathrm{~V}$ ), does not guarantee an OVPM-trigger in one of the other sampling points. The required voltage for an OVPM-trigger is much higher than the voltage required for a symbol change. Thus, less symbol changes are captured by the OVPM.

Hence, the first trade-off is having an increased data rate versus having more grip on capturing the symbol changes with the OVPM. For NRZ-L, a symbol change is usually caught at another sampling point by the OVPM. This is much less the case for PAM-4, but the system operates at a double bit rate.

\section{B. Correct Data (EFD and SCO)}

The EFD category decreases the most in this assessment. This category goes from $50.64 \%$ to $10.02 \%$ when changing to PAM-4. Furthermore, Figs. 2 and 3 clearly show a significant rise in the proportion of correctable corruptions (SCO). This category increases from $1.44 \%$ to $21.93 \%$.

This significant difference was to be expected. By lowering the noise margin between the code voltages, higher chances exist of introducing bit flips in the data transmission. As mentioned, the noise margin drops from 0.25 to $0.083 \mathrm{~V}$.

In the best scenario, all packets that were error-free in NRZ-L are transformed into correctable packets of PAM-4. However, this is not the case. More packets from the EFDcategory are corrupted than can be corrected: EFD lowers by about $40 \%$, while the SCO rises by about $20 \%$. When considering the availability, this a significant trade-off for the obtained double bit rate. There is $20 \%$ less data correctly arriving at the receiver. In this paper, availability is defined 
as the system behaving correctly. It is thus the sum of EFD and SCO.

At this point, the double data rate must be taken into account to determine if PAM-4 is worth using it. Assuming a channel with a symbol rate of 1 Gigabaud. For the NRZ-L, there are 12 bits in a data word, encoded as 12 symbols, providing 83.3 million data words per second. Of those, $52.08 \%$ are on average correct, yielding 43.2 million correct words per second. For PAM-4, the same calculation yields: 166.3 million data words per second, of which 53.25 million data words per second are correct. Thus, from an availabilitys point of view, the use of a PAM-4 coding is justified.

\section{Incorrect Data (FCO and FN)}

The FCO category shows a major increase between NRZ-L and PAM-4: from $1.05 \%$ to $34.57 \%$ of the time. In terms of False Negatives, the PAM-4 shows a decline: from $0.228 \%$ to $0.157 \%$. Even though this is only a difference of $0.071 \%$, it is lower for PAM-4. Also, since reducing FNs is the main focus of the research, more weight is given to that reduction.

For FCO, the cause closely relates to SCO: the noise margin is reduced, allowing more symbol flips to pass through without the OVPM being triggered. The more the ECC is needed, the more it can fail while attempting to correct the induced bit flips.

In terms of safety, the FCO category is not the worst; at least a notification is passed to the system that a correction attempt was made. In essence, this category relies on upper layers to resolve the problem of incorrectly received data.

Alongside the FCO category, the FN category is of major importance in this paper. A small decline in the rate of False Negatives is observed. However, the same nuance as in Section III.B is needed: the double data rate. The same channel with a symbol rate of $1 \mathrm{GBd}$ is considered. For NRZ-L line coding, this yields 190,250 False Negatives per second under such a harsh EMI disturbance. For a PAM-4, this number goes up to 263,333 False Negatives per second. Thus, in terms of safety under such harsh EMI conditions, the use of PAM-4 does not seem to be recommended. It has a much higher absolute rate of False Negatives (whereas the percentages would suggest otherwise). In absolute numbers of False Negatives per second, PAM- 4 is about $38.41 \%$ worse.

Upon investigating the results of the experiments, it is found that nearly all False Negatives are received as either all-zero, or all-one data, regardless of the originally sent data. A very effective strategy in reducing False Negatives is to not allow this code word to be valid. In that case, when 0000 or 1111 would be received as data, the receiver knows this is a False Negative. It no longer considers those data words to be valid. Although some exceptions will still be present as a False Negative, this already significantly reduces the rate of False Negatives. Also, this strategy is easy to implement, e.g. with bit stuffing. The reduction in allowed code words is expected to be recovered by the increased data rate. Exact calculations on this topic are not the scope of this paper.

\section{General Assessment}

For the general assessment, the prioritization of the system is important. Is this system designed for availability or safety?

When the former is true, then choosing PAM-4 is a good option. The combination for availability (EFD and SCO) yields higher absolute throughput for PAM-4. This is mainly due to the double data rate, which overcomes the problems introduced by the lowered noise margin. An ECC is also recommended, since the lowered noise margin will result in an increased number of symbol flips to be induced on the channel. Note that the triplication used in this paper was unable to cope with the increased number of bit flips in the code words.

The second design constraint can be safety. PAM-4 has the advantage of a lower rate of False Negatives, when observed in percentages. However, by using a specific example with an actual symbol rate, the PAM-4 is found to be worse than the NRZ-L line coding. In safety-critical applications, the NRZ-L line coding is preferred instead of a PAM-4.

\section{CONCLUSIONS}

This paper builds on previous research, where a communication network was protected by Error Correction Codes (ECCs). However, these codes implemented a high overhead to counteract the rate of False Negatives. This paper attempted to lower the transmission overhead introduced by ECCs, while maintaining the same resiliency against harsh environments. In order to reduce the overhead, a PAM-4 line coding was introduced to replace the originally used NRZ-L line coding. It is concluded that PAM-4 can be used in harsh environments, but does not yield the same safety-rating as a NRZ-L coding. Furthermore, the introduced ECC is unable to counteract the bit flips induced by the reduced noise margins. For availability this line coding is a valid choice, but is found inadequate for safety-critical applications.

\section{REFERENCES}

[1] J. Van Waes, J. Lannoo, A. Degraeve, D. Vanoost, D. Pissoort, and J. Boydens, "Effectiveness of cyclic redundancy checks under harsh electromagnetic disturbances," in Proc. Int. Symp. Electromagnetic Compatibility - EMC EUROPE, Sep. 2017.

[2] J. Van Waes, J. Lannoo, J. Vankeirsbilck, A. Degraeve, J. Peuteman, D. Vanoost, D. Pissoort, and J. Boydens, "Effectiveness of hamming single error correction codes under harsh electromagnetic disturbances," in Proc. Int. Symp. Electromagnetic Compatibility - EMC EUROPE, 2018.

[3] J. Van Waes, J. Vankeirsbilck, J. Lannoo, D. Pissoort, and J. Boydens, "Effectiveness of data triplication in harsh electromagnetic environments," in Proc. Int. Symp. Electromagnetic Compatibility - EMC EUROPE, 2018.

[4] J. Van Waes, D. Vanoost, J. Vankeirsbilck, J. Lannoo, D. Pissoort, and J. Boydens, "Resilience of error correction codes against harsh electromagnetic disturbances: Fault mechanisms," IEEE Transactions on Electromagnetic Compatibility, pp. 1-11, 2019.

[5] J. Van Waes, J. Lannoo, J. Vankeirsbilck, D. Vanoost, D. Pissoort, and J. Boydens, "Resilience of error correction codes against harsh electromagnetic disturbances: Fault elimination for triplication-based error correction codes," IEEE Transactions on Electromagnetic Compatibility, 2019, accepted Oct 17, 2019.

[6] J. Van Waes, J. Vankeirsbilck, J. Lannoo, D. Vanoost, D. Pissoort, and J. Boydens, "Complementary fault models for assessing the effectiveness of hamming codes," in IEEE International Scientific Conference Electronics (ET), 2019, accepted. 
[7] J. Lannoo, J. Van Waes, A. Degraeve, D. Vanoost, J. Boydens, and D. Pissoort, "Effectiveness of time diversity to obtain EMI-diverse redundant systems," in Proc. Int. Symp. Electromagnetic Compatibility (EMC EUROPE), Aug. 2018, pp. 288-292.

[8] F. Vanhee, D. Pissoort, J. Catrysse, G. A. E. Vandenbosch, and G. G. E. Gielen, "Efficient reciprocity-based algorithm to predict worst case induced disturbances on multiconductor transmission lines due to incoming plane waves," IEEE Transactions on Electromagnetic Compatibility, vol. 55, no. 1, pp. 208-216, Feb. 2013.

[9] D. A. Hill, "Plane wave integral representation for fields in reverberation chambers," IEEE Transactions on Electromagnetic Compatibility, vol. 40, no. 3, pp. 209-217, Aug. 1998.

[10] M. Magdowski, S. V. Tkachenko, and R. Vick, "Coupling of stochastic electromagnetic fields to a transmission line in a reverberation chamber," IEEE Transactions on Electromagnetic Compatibility, vol. 53, no. 2, pp. 308-317, May 2011.

[11] J. Shi, "ESD characteristics of ggnmos device in deep sub-micron cmos technology," 2016 International Conference on Audio, Language and Image Processing (ICALIP), pp. 327-331, 2016. 